\title{
Expansion of MT Neurons Excitatory Receptive Fields during Covert Attentive Tracking
}

\author{
Robert Niebergall, ${ }^{1,2}$ Paul S. Khayat, ${ }^{3}$ Stefan Treue, ${ }^{2,4}$ and Julio C. Martinez-Trujillo ${ }^{1}$ \\ ${ }^{1}$ Cognitive Neurophysiology Laboratory, Department of Physiology, McGill University, Montreal, Quebec H3G 1Y6, Canada, ${ }^{2}$ Cognitive Neuroscience \\ Laboratory, German Primate Center, 37077 Goettingen, Germany, ${ }^{3}$ Department of Psychology, University of Montreal, Montreal, Quebec H3C 3J7 Canada, \\ and ${ }^{4}$ Bernstein Center for Computational Neuroscience Goettingen, 37073 Goettingen, Germany
}

Primates can attentively track moving objects while keeping gaze stationary. The neural mechanisms underlying this ability are poorly understood. We investigated this issue by recording responses of neurons in area MT of two rhesus monkeys while they performed two different tasks. During the Attend-Fixation task, two moving random dot patterns (RDPs) translated across a screen at the same speed and in the same direction while the animals directed gaze to a fixation spot and detected a change in its luminance. During the Tracking task, the animals kept gaze on the fixation spot and attentively tracked the two RDPs to report a change in the local speed of one of the patterns' dots. In both conditions, neuronal responses progressively increased as the RDPs entered the neurons' receptive field (RF), peaked when they reached its center, and decreased as they translated away. This response profile was well described by a Gaussian function with its center of gravity indicating the RF center and its flanks the RF excitatory borders. During Tracking, responses were increased relative to Attend-Fixation, causing the Gaussian profiles to expand. Such increases were proportionally larger in the RF periphery than at its center, and were accompanied by a decrease in the trial-to-trial response variability (Fano factor) relative to Attend-Fixation. These changes resulted in an increase in the neurons' performance at detecting targets at longer distances from the RF center. Our results show that attentive tracking dynamically changes MT neurons' RF profiles, ultimately improving the neurons' ability to encode the tracked stimulus features.

\section{Introduction}

In many situations, humans and other primates must attentively track moving objects while keeping gaze stationary. It has been suggested that this ability relies on covert attention (Cavanagh and Alvarez, 2005). A single or multiple moving spotlights of attention may highlight tracked objects and enhance their neuronal representations in visual cortex. Supporting this hypothesis, previous studies of multiple-object tracking (MOT) in humans have demonstrated that visual event-related potentials (ERPs) and BOLD signals evoked by attentively tracked objects are enhanced relative to those evoked by distracters (Culham et al., 1998; Drew et al., 2009; Howe et al., 2009; Doran and Hoffman, 2010). The mechanisms of these effects at the level of single visual neurons remain poorly understood.

Received June 6, 2011; revised Sept. 5, 2011; accepted Sept. 8, 2011.

Author contributions: R.N., S.T., and J.C.M.T. designed research; R.N. and P.S.K. performed research; R.N., P.S.K., and J.C.M.T. contributed unpublished reagents/analytic tools; R.N. and J.C.M.T. analyzed data; R.N. and J.C.M.T. wrote the paper.

This work was supported by grants to J.C.M.T. from the Canada Research Chairs program, the Canadian Foundation for Innovation, the Canadian Institutes for Health Research, and the ELL foundation. P.S.K. was supported by a postdoctoral fellowship from the National Science and Engineering Research Council of Canada. S.T. and R.N. were supported by the Bernstein Center of Computational Neuroscience Göttingen (Grants 01 GQ0433 and 01GQ1005C), the Bundesministerium für Bildung und Forschung and the Deutsche Forschungsgemeinschaft Collaborative Research Center 889 "Cellular Mechanisms of Sensory Processing". R.N. was also supported by a doctoral fellowship from the Deutscher Akademischer Austausch Dienst.

This article is freely available online through the J Neurosci Open Choice option.

Correspondence should be addressed to Dr. Julio C. Martinez-Trujillo, Cognitive Neurophysiology Laboratory, Department of Physiology, McGill University, 3655 Promenade Sir William Osler, Montreal, Quebec H3G 1Y6, Canada. E-mail: julio.martinez@mcgill.ca.

DOI:10.1523/JNEUROSCI.2822-11.2011

Copyright $\odot 2011$ the authors $\quad 0270-6474 / 11 / 3115499-12 \$ 15.00 / 0$
A recent electrophysiological study in monkeys recorded the responses of neurons in visual area V4 during a MOT task and demonstrated that when a tracked object stops and remains stationary inside a neuron's receptive field (RF), responses are stronger than those evoked when the object is ignored (Sundberg et al., 2009). This result agrees with previous reports of enhancement in the responses of visual neurons to stationary behaviorally relevant objects (Moran and Desimone, 1985; Spitzer et al., 1988; Treue and Maunsell, 1996; McAdams and Maunsell, 1999; Reynolds et al., 1999; Seidemann and Newsome, 1999; Treue and Martinez-Trujillo, 1999). One issue that remains unclear is how attention modulates responses of visual neurons to tracked objects that dynamically change position in space.

One possibility is that during attentive tracking of a moving object, the profile of a visual neuron's RF undergoes dynamic changes similar to those reported during tasks where attention is directed to stationary objects (Connor et al., 1996; Womelsdorf et al., 2006; Anton-Erxleben et al., 2009). For example, it is possible that during attentive tracking, a neuron multiplicatively increases the response to a passing tracked stimulus over the entire RF excitatory area, producing a homogenous expansion of the RF. Alternatively, the neuron may differentially increase the responses to the passing stimulus across different RF regions, e.g., larger increases at the center and smaller in the periphery, or vice versa. The former would produce a sharpening of the RF profile. The latter would resemble a nonlinear expansion of the RF excitatory border, with larger increases in response close to the border relative to the center. 
Here, we tested these ideas by recording single-neuron activity in extrastriate visual area MT of two rhesus monkeys to stimuli passing through the neurons' RFs during two different tasks: (1) when the animals attentively tracked the stimuli (Tracking) and (2) when they ignored the stimuli and attended elsewhere (Attend-Fixation). We found that during Tracking, MT neurons increased their responses to the stimuli passing across their RF, with larger increases within regions away from the RF center. Such increases were accompanied by a progressive decrease in response variability during Tracking as the tracked stimuli approached the RF center. These changes improved the neurons' ability to detect and encode the features of the stimuli during Tracking relative to Attend-Fixation.

\section{Materials and Methods}

Two adult male rhesus monkeys (Macaca mulatta, 5.5 and $7.2 \mathrm{~kg}$ ) participated in the experiments. All procedures complied with the Canadian Council of Animal Care guidelines and were preapproved by the McGill University Animal Care Committee.

\section{Apparatus and stimuli}

A custom-written software running on an Apple G4 computer controlled the stimulus presentation as well as the recording of eye positions and behavioral responses. Stimuli were back-projected onto a screen by a video projector (WT610; NEC Display Solutions of America) at a resolution of $1024 \times 768$ pixels and a refresh rate of $85 \mathrm{~Hz}$. The animals sat in a primate chair in front of the screen at a viewing distance of $57 \mathrm{~cm}$. The stimuli were moving random dot patterns (RDPs) composed of small bright dots $\left(\right.$ dot size $=0.01$ degrees $^{2}$, dot density $=5$ dots per degrees ${ }^{2}$ ) moving behind circular apertures on a dark background $\left(0.02 \mathrm{~cd} / \mathrm{m}^{2}\right)$. The dots could be either green $\left(12.8 \mathrm{~cd} / \mathrm{m}^{2}\right)$ or red $\left(14.6 \mathrm{~cd} / \mathrm{m}^{2}\right)$ and moved with $100 \%$ coherence. When they crossed the aperture's border they were replotted on the opposite side. The diameter of each RDP was adjusted to be $\sim 1 / 3$ of the RF diameter. Eye positions were recorded using a video-based eye tracking system (Eye Link 1000, SR Research) with a sampling frequency of $200 \mathrm{~Hz}$.

\section{Task}

At the beginning of a trial, the animals directed and maintained gaze on a white fixation spot (FS) in the center of the screen and pressed a button to initiate the stimulus presentation. After $590 \mathrm{~ms}$, two red or green RDPs appeared outside the recorded neuron's RF (Fig. 1A). They translated across the screen following parallel trajectories at a constant velocity of $3.5 \%$ and crossed the RF. Their initial position was either proximal to the FS, and hence they translated toward the periphery (Fig. 1 A, outward); or distal to the FS and they translated toward the center of gaze (Fig. $1 \mathrm{~A}$, inward).

The animals were trained in two task conditions. When the FS turned from white to light gray, they had to detect a change in the FS luminance while ignoring the RDPs (Fig. $1 B$, Attend-Fixation). When the FS color turned to the color of the RDPs (red or green), they had to keep fixating and detect a brief $(110 \mathrm{~ms})$ change in the local speed of the dots in one of the patterns. The speed change occurred with equal probability $(0.5)$ in either pattern (Fig. $1 B$, Tracking). The color variations of the RDPs were meaningless in the context of the present study, but were necessary for a second experiment run in parallel. However, there were no differences in response between stimuli of different colors, as tested by recording the responses to a single stimulus positioned inside the RF while varying its color from trial to trial ( $p=0.79$, paired $t$ test, data not shown). The patterns' positions were always isoeccentric with respect to the FS. Dots in the two RDPs could locally move in the neuron's preferred or antipreferred direction. The latter was defined as the direction $180^{\circ}$ from the preferred direction (Treue and Martinez-Trujillo, 1999). Within a trial, the dots always moved in the same direction.

During a recording session, Attend-Fixation and Tracking trials with RDPs of different directions and colors (i.e., both patterns red/ green and moving in the preferred/antipreferred direction) were randomly interleaved. The speed change intensity was chosen in such a way that the proportion of correct detections in the majority of the sessions was $75 \%$ or higher. All changes occurred at a random time $(820-5060 \mathrm{~ms})$ from trial onset, challenging the animals to sustain attention on the targets throughout the trial. Releasing the button within 150-650 ms from target change onset was considered a hit and rewarded with juice.

\section{Surgical procedures and recordings}

In each animal, a titanium head post (custom made) and a Cilux plastic recording chamber (diameter $=20 \mathrm{~mm}$; Crist Instruments) were surgically implanted under general anesthesia. The chambers were positioned over a craniotomy in the parietal bone, providing dorsal access to the superior temporal sulcus. Localization of area MT was conducted using postsurgical structural magnetic resonance imaging (Siemens 3T Trio MR scanner) (for MRI images and stereotactic coordinates of recording sites, see Khayat et al., 2010a).

In each session, guide tubes were used for transdural penetrations. Epoxylite-insulated tungsten electrodes (diameter $=100-125 \mu \mathrm{m}$, impedance $=1-4 \mathrm{M} \Omega ; \mathrm{FHC}$ ) were inserted into area MT through the guide 
tube using an electrical microdrive (NAN Instruments). Action potentials were isolated based on the online display of neural signals using Plexon software and recording equipment (Plexon), and an analog-digital oscilloscope (Hameg Instruments). Spikes were amplified and filtered between $250 \mathrm{~Hz}$ and $10 \mathrm{kHz}$ before being digitized and stored at $40 \mathrm{kHz}$. A neuron was determined to be from MT based on its response properties to moving RDPs of different directions and speeds, the location and size of its RFs, and the position of the electrode relative to the superior temporal sulcus (Khayat et al., 2010a).

\section{Data analysis}

All the analyzed neural data were obtained from hit trials and were truncated at the time of the target change. On average $( \pm S D)$, the animals correctly performed $29( \pm 8)$ trials per stimulus configuration and condition. For each trial, we computed a spike density function (SDF) by convolving each spike with a Gaussian kernel ( $\sigma=25 \mathrm{~ms})$.

Since target changes occurred at different times during the trial, the spatial distance covered by the translating stimuli in each trial was variable. This was done to encourage the animal to attend to the stimulus during the whole trial length. The data used in the analysis comprised the time/space where a minimum of five trials where available. The length of the data in the fixation condition matched the length of the data in the tracking condition. In both conditions, individual trials were binned according to the length of the available data. A single bin was equivalent to (length of data)/10. This yielded an average bin size $( \pm$ SD) of 344 $( \pm 59) \mathrm{ms}$ (equivalent to $1.2 \pm 0.2^{\circ}$ of visual angle). The firing rates of individual trials were subsequently pooled to obtain an average SDF per bin.

Classification of neurons. We recorded 157 single neurons during 147 recording sessions (in some sessions, two neurons with similar RF locations and feature selectivity were simultaneously isolated from the same electrode). The neurons' RF sizes were mapped on-line with a moving bar or a stationary RDP attached to the cursor. To account for differences in RF size and as part of a second experiment, the RDPs were positioned close together in approximately half of the recording sessions and farther apart in the other half. For each neuron, we determined whether the RDPs crossed the excitatory region of the RF and evoked a response by fitting the following Gaussian equation to the binned average $\operatorname{SDF}\left(R_{\text {avg }}\right)$ in both configurations:

$$
R_{\text {avg }}=B+H \times e\left[(C-R(p))^{2} / W^{2}\right],
$$

where $B$ represents the neuron's firing rate when the RDPs are outside the RF; $H$ describes the height of the curve; $R(p)$ corresponds to the response at position $p$; $C$ represents the location of the peak response, which approximates the RF center; and $W$ provides an estimate of the excitatory $\mathrm{RF}$ region extent. Data were fitted using the Matlab optimization toolbox (Matworks) and the least-squares fitting method (Treue and MartinezTrujillo, 1999). Neurons were categorized as modulated by the RDPs when the correlation coefficient $\left(R^{2}\right)$ of one of the fits (in either Tracking or Attend-Fixation) exceeded 0.8 and the $R^{2}$ of the fit in the other condition was $>0.5$. This procedure was applied to both outward and inward trials, which were analyzed separately to detect potential effects of the RDPs' motion direction across the screen. A total of 134 (70 outward and 64 inward) neurons matched the criterion. In addition to the Gaussian fit, we computed for each neuron a direction selectivity index (DSI) in Attend-Fixation trials:

$$
\mathrm{DSI}=\left(R_{\text {pref }}-R_{\text {antipref }}\right) /\left(R_{\text {pref }}+R_{\text {antipref }}\right),
$$

where $R_{\text {pref }}$ and $R_{\text {antipref }}$ are the mean responses to the preferred and antipreferred RDP directions in each response bin, respectively.

Border expansion analysis. The peak of the Gaussian fit during AttendFixation was defined to be the RF center and was used as a reference to measure the distance to the point along the abscissa were the AttendFixation fit reached half of the peak response. In the following sections, this distance will be termed $\mathrm{DFR}_{50}$. The DFR ${ }_{50}$ during Tracking was also determined with respect to the point of half-height during AttendFixation. For the population analysis, changes in the position of the RF excitatory borders during Tracking were expressed as percentage of the $\mathrm{DFR}_{50}$ during Attend-Fixation trials.
Analysis of fitting parameters. Differences in parameters of the fits between Attend-Fixation and Tracking were quantified by computing parameter modulation indices (PMIs) of the form:

$$
\text { PMI }=\left(P_{\text {tracking }}-P_{\text {Pattend-fixation }}\right) /\left(P_{\text {tracking }}+{ }_{\text {Pattend-fixation }}\right),
$$

where $P$ represents the parameter. Positive PMIs indicate higher parameter values during Tracking and negative PMIs indicate higher parameter values during Attend-Fixation. A PMI of zero indicates no differences. A similar index was computed to analyze differences in maximum firing rates between the two conditions.

Since the width of the Gaussian fit is a critical measurement in this study, a second analysis was applied to determine whether changes in width significantly improve the fits in the Tracking condition. First, the data in the Attend-Fixation condition were fitted with the Gaussian model described above (Eq. 1). Second, two different models were fitted to the Tracking data. In the first model, the width was allowed to vary. In the second model, the width parameter was fixed to the width value determined during Attend-Fixation. We assessed the goodness of fit of the two models using the Akaike information criterion (AIC) (Akaike, 1974):

$$
\mathrm{AIC}=n \times \ln \left(\frac{\mathrm{SSE}}{n}\right)+2 k
$$

where $n$ represents the number of data points, $k$ the number of model parameters, and SSE is the sum of squared errors. The AIC is a statistical measure used to compare the goodness of fit of models with different numbers of free parameters. As can be derived from the formula, the model that best describes the data is the one with the lowest AIC value.

The difference in AIC between the first (varying width) and the second (fixed width) model was computed using the following formula:

$$
\Delta \mathrm{AIC}=\mathrm{AIC}_{\text {varying }}-\mathrm{AIC}_{\text {fixed }}
$$

If $\Delta \mathrm{AIC}$ is negative, the difference in sum squared error between models is greater than that expected based on the difference in the number of parameters ( 4 vs 3 ), implying that the model with varying width provides a better description of the data. For each neuron, $\triangle$ AIC was computed and subsequently averaged across neurons for statistical analysis using $t$ tests.

Analysis of nonlinear RF expansion. To test whether tracking causes a nonlinear receptive field expansion, we applied a second method that was independent of the Gaussian model fits. For each neuron, we determined the location of the peak response during Attend-Fixation (the RF center). In each trial, data before stimulus onset (baseline response) and after the time of the peak response were truncated. The remaining part of singletrial firing rates was binned according to the length of the available data. To compute population responses, average SDFs of each neuron were normalized to the peak response during fixation and pooled across neurons. In addition, a response modulation index (RMI) was computed for each neuron and response bin, as follows:

$$
\mathrm{RMI}=\left(R_{\text {tracking }}-R_{\text {att-fix }}\right) /\left(R_{\text {tracking }}+R_{\text {att-fix }}\right)
$$

where $R_{\text {tracking }}$ represents the responses obtained during Tracking and $R_{\text {att-fix }}$ those obtained during Attend-Fixation (Khayat et al., 2010). RMIs of individual neurons were pooled for statistical analysis. Note that the RMI may magnify the differences in response between low spiking neurons. However, since we used responses to the preferred direction, most of our neurons gave a robust response to the stimulus when entering the RF borders.

Signal-detection analysis. To determine each neuron's ability to detect the RDPs entering its RF, we applied a signal detection analysis. Different from the $\mathrm{DFR}_{50}$ and the tuning curve parameters analyses, this analysis incorporates the neuron's mean firing rate across trials as well as the intertrial rate variability (Thompson et al., 1996). Additionally, this analysis is also independent of the Gaussian fitting procedures described in Classification of neurons, above. We computed receiver operator characteristic (ROC) curves for each neuron using distributions of firing rates at trial onset, and at successive time intervals after trial 
onset (see below). We then computed the area under the ROC curve (auROC), which represents the probability that an ideal observer can reliably identify the separation between the two firing-rate distributions. In the present context, this analysis indicates at what distance from the RF center the translating stimuli were detected.

For each trial and condition, we computed the average SDF during the first $20 \mathrm{~ms}$ after the onset of the RDPs, i.e., when they were located outside the RF boundaries. Relative to this baseline period, we calculated the auROC curves using a 20 $\mathrm{ms}$ window and $1 \mathrm{~ms}$ steps. An auROC value of 0.5 indicates that the firing rates during a given period cannot be distinguished from baseline. A value $>0.5$ indicates that responses during the analyzed period are greater than baseline. A value $<0.5$ indicates that responses are smaller than baseline.

For each condition, we obtained auROC curves that typically increased as the RDPs approached the RF center. To describe this distance-dependent effect, we binned the auROC data of each condition into 10 bins of similar size and fitted the resulting set of data points with a Weibull function, as follows:

$$
W(p)=\gamma-(\gamma-\delta) \times \exp \left[-(t / \alpha)^{\beta}\right],
$$

A

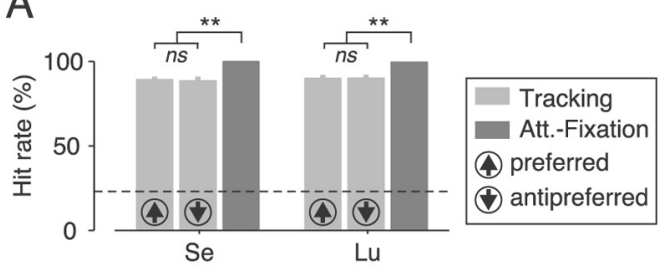

B
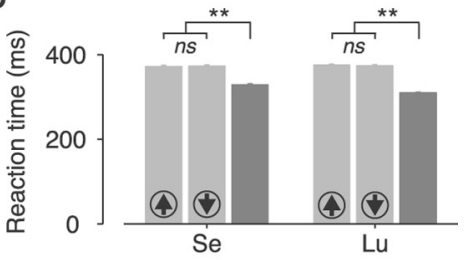

C
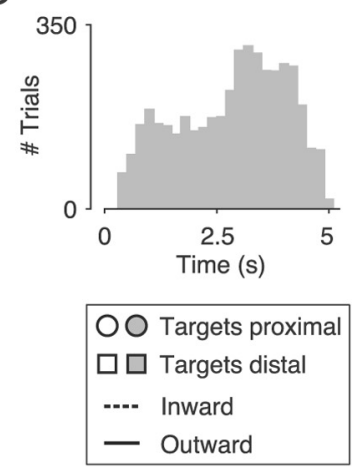

D
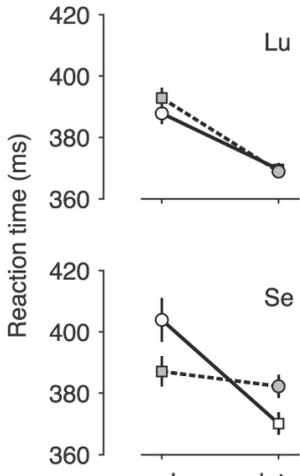

Lu

$E$

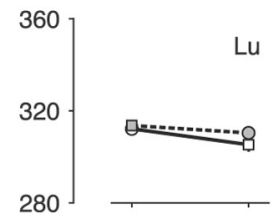

Se
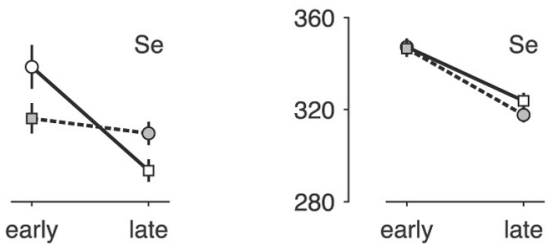

Target change occurrence

Figure 2. Behavioral performance. $A$, Average hit rate during Tracking (light gray) and Attend-Fixation (dark gray) of monkeys $\mathrm{Se}(n=64)$ and $\mathrm{Lu}(n=83) .{ }^{* *} p<0.0001$, Wilcoxon signed rank test. $\boldsymbol{B}$, Average reaction times. Colors as in $\boldsymbol{A} .{ }^{* *} p<0.0001$; unpaired $t$ test. $n s$, Nonsignificant. $\boldsymbol{C}$, Distribution of target change times. $\boldsymbol{D}, \boldsymbol{E}$, Average reaction times during tracking $(\boldsymbol{D})$ and fixation (E) for early and late target changes. Error bars indicate $95 \%$ confidence intervals. Att., Attend. where $W(p)$ is the value of the auROC when the translating RDPs were at position $p$ (measured in degrees from their initial position), $\alpha$ is the time at which the function reaches $64 \%$ of its full growth, $\beta$ is the slope, $\gamma$ is the upper asymptote, and $\delta$ is the lower asymptote.

The position at which the RDPs entered the RF was defined as the position $p$ where $W(p)$ reached the threshold criterion of 0.75 (Thompson et al., 1996). The distance between the RDPs initial position and this point was termed target-detection distance (TDD) and can be used as an estimate of the RF border (see Fig. 7A). Neurons that failed to reach criterion in both the Attend-Fixation and Tracking conditions were removed from the analysis $(n=12)$. For each neuron, we computed the difference in TTD, as follows:

$$
\Delta \mathrm{TDD}=\mathrm{TDD}_{\text {tracking }}-\mathrm{TDD}_{\text {fixation }}
$$

Negative values indicate that the approaching targets were detected at a shorter distance from their initial position during Tracking relative to Attend-Fixation and positive values indicate the contrary.

Fano factor analysis. As a measure of trial-to-trial variability, we computed the Fano factor, the ratio of the variance of the spike counts across trials divided by the mean of the spike counts (Mitchell et al., 2007, 2009; Cohen and Maunsell, 2009). This analysis was conducted on raw spike counts rather than on single-trial SDFs. For each neuron and both conditions, the number of spikes in a counting window of size $12 \mathrm{~ms}$ was determined for non-overlapping windows over the length of each trial. The results were used to compute the mean and variance across trials in each window. To pool data from different neurons, the length of the available Fano factor data was divided into 10 response bins, and the average Fano factor for each bin was computed. To assess the effect of attention, we computed a Fano factor modulation index (FFMI) for each neuron and response bin, using the following:

$$
\text { FFMI }=\left(\mathrm{FF}_{\text {tracking }}-\mathrm{FF}_{\text {att-fix }}\right) /\left(\mathrm{FF}_{\text {tracking }}+\mathrm{FF}_{\text {att-fix }}\right),
$$

where $\mathrm{FF}_{\text {tracking }}$ represents the Fano factor obtained during tracking and $\mathrm{FF}_{\text {att-fix }}$ represents the Fano factor obtained during Attend-Fixation.

Performance. For each recording session, the average percentage of correct change detections (hit rate) was computed. Failures to release the button within the response time window (150-650 ms after the target change occurred) were considered errors. Fixation errors (trials terminated because the animal's gaze deviated from the FS) were not included in the performance computation. The chance hit rate was 23\% [computed by dividing the response time window $(500 \mathrm{~ms})$ by the average length of the trial period where a target event could occur $(2180 \mathrm{~ms})]$. Reaction times were defined as the time between the onset of the target change and button release. Since we were interested in covering a large portion of the RF, the distribution of possible target change times was biased toward longer times during the trial (Fig. 2C).

To assess changes in task difficulty over time during Attend-Fixation and over time and across space during Tracking, we divided the trials of each recording session into two response bins. The first one contained all reaction times of trials where target changes occurred within the time between the earliest possible change and the median of all change event times (early). The second one contained all reaction times of trials where changes occurred between the median and the latest possible change time (late). Analyses of hit rates were conducted using nonparametric tests, and analyses of reaction times were conducted using parametric tests.

Eye position measurements. Eye movements were measured using a videobased eye tracking system (Eye Link 1000, SR Research). Horizontal and vertical eye position signals were fed into the stimulus-presentation computer and converted into degrees of visual angle according to a calibration procedure conducted at the beginning of each experimental session. From these signals, we computed average eye positions for each trial and condition. The monkeys could only start a trial if their gaze was within a $1^{\circ}$ radius from the FS center. If at any time during a trial the animal's gaze moved outside the fixation window, the trial was aborted without reward (Khayat et al., 2010a).

\section{Results}

\section{Behavioral performance}

To determine whether the animals correctly performed the tasks, we quantified their hit rates and reaction times during both conditions across all recording sessions. During Tracking, the responses to the preferred and antipreferred RDPs were analyzed 
separately. Hit rates of both animals were considerably above chance (23\%; Fig. $2 A$, dashed line) in all conditions ( $p<0.0001$, Wilcoxon signed rank tests). The average hit rates in response to the preferred and antipreferred directions of the local dots in the RDPs were similar (monkey Se: $n=64$, preferred $=89 \%$, antipreferred $=88 \%, p=0.73$; monkey Lu: $n=83$, preferred $=90 \%$, antipreferred $=90 \%, p=0.88$, Wilcoxon paired rank sum test for paired samples; Fig. $2 A$, light gray) and were significantly lower than those during Attend-Fixation (monkey Se: mean = 99\%, $p<0.0001$; monkey Lu: mean $=99 \%, p<0.0001$, Wilcoxon rank sum test for paired samples; Fig. $2 A$, dark gray).

Both animals' average reaction times across sessions followed a similar trend (Fig. 2 B). During Tracking, they were similar for the two directions of the RDPs local dots (monkey Se: preferred $=371 \mathrm{~ms}$, antipreferred $=372 \mathrm{~ms}, p=0.8$; monkey Lu: preferred $=375 \mathrm{~ms}$, antipreferred $=373 \mathrm{~ms}, p=0.65$, unpaired $t$ test; Fig. $2 B$, light gray) and were longer than those during Attend-Fixation (monkey Se: mean $=328 \mathrm{~ms}, p<0.0001$; monkey Lu: mean $=309 \mathrm{~ms}, p<0.0001$; Fig. 2 B, dark gray). These results suggest that the local dot motion of the RDPs was irrelevant for the animals' tracking performance, and that they performed better during Attend-Fixation relative to Tracking trials. Note that during Attend-Fixation trials, the animals detected a change in contrast; and during Tracking trials, they detected a change in the speed of the RDPs. Thus, the difference in performance may simply illustrate different task demands. Nevertheless the main point here is that in one condition, the animals mainly attended to the fixation point and, in the other, to the translating RDPs.

We additionally assessed whether the animals preferentially attended to one of the two RDPs during Tracking by comparing reaction time distributions corresponding to speed changes in each target within a session using unpaired $t$ tests (significance level: $p=0.05$ ). We found that the two distributions were significantly different in only six of the 147 sessions (data not shown). Thus, in individual sessions, the animals reacted equally fast to changes in either pattern, suggesting that they divided attention between both RDPs.

In our experimental design, at least two different variables acting in different ways could have influenced task difficulty during a trial: the RDPs eccentricity and the trial duration. In the former case, one might expect performance to be better at small eccentricities when the RDPs are closer to the fixation point. Thus, in the outward condition, reaction times should be shorter in short trials (when RDPs are closer to the fixation point) relative to long trials (when RDPs are more eccentric). For the inward condition, however, the effect should be reversed, i.e., reaction times should be longer for short trials (when RDPs are in the periphery) and shorter for long trials (when RDPs are closer to the fixation point). For the case of trial duration, if performance were primarily driven by the probability of occurrence of the target change (hazard function), one would expect reaction times to be shorter for long trials independently of the RDPs' position in the visual field. This is a reasonable assumption because in our experiment, the distribution of possible target changes was biased toward longer trials (to maximize the RF area covered by the RDPs), as can be seen in the histogram displaying target change times in Figure 2C.

We tested which one of the two factors primarily determined performance by dividing the reaction times of each monkey into two groups. The first one (early) included the reaction times during trials when the target change occurred before the median target change time of a recording session. The second group (late) was comprised of reaction times during trials where the target changes occurred after the median target change time. In outward trials, early changes represent targets that are closer to the fixation spot (proximal) and late trials represent targets that are in the periphery (distal). For inward trials, this relationship reverses. Figure $2 D$ shows the average reaction times of the early and late target changes during Tracking for both the outward (dotted line and gray symbols) and the inward (solid line and white symbols) group of trials. For both monkeys (Fig. 2 D, monkey Lu, top; monkey Se, bottom), we computed a two-way ANOVA with target change time (early and late) and translating direction (inward and outward) as factors. Reaction times were significantly shorter for the late target changes in both animals $(p=0)$, indicating that reaction times were mainly influenced by the time at which target changes occur. Neither monkey showed a significant effect of translation direction $(\mathrm{Lu}, p=0.15$; Se, $p=$ 0.39 ). However, interaction effects were significant in both animals (Lu, $p=0.049$; Se, $p=0$ ).

We conducted a similar analysis during Attend-Fixation trials. Although here the target eccentricity will not play a role because the animals were attending to the fixation spot, we divided the trials into outward and inward according to the stimulus configuration. We observed an effect of early and late, as in the previous analysis, with shorter reaction times in late trials in both animals $(p<0.05$, two-way ANOVA). These results suggest that task difficulty was not greatly influenced by the RDPs position in the visual field, but rather by the probability of the target change over time. We can conclude that the effects isolated for the two translation directions of the RDPs, as well as during the AttendFixation task, are similarly affected by task timing.

\section{Analysis of neuronal responses}

We recorded responses of 157 MT neurons to the stimulus configuration illustrated in Figure $1 A$. For the analysis, we selected only neurons showing a clear response modulation as a function of the RDP position that could be modeled by a Gaussian function (see Materials and Methods, above). Figure $3 A$ shows the responses of an example neuron in the two conditions to RDPs translating outwards. Attend-Fixation responses (Fig. $3 A$, white circles) change as a function of the RDPs' position on the screen (i.e., distance from RDP onset position). They depart from a baseline level, progressively increase as the RDPs enter the RF, peaking at its center, and progressively decrease as the RDPs move away from the center. During Tracking (Fig. $3 A$, gray circles), the neurons exhibited a similar response profile. However, compared with Attend-Fixation, responses depart from baseline earlier and reach a higher maximum at the RF center. In both conditions, the responses are well fit by Gaussian functions (Fig. $3 A$, solid and dashed lines). The same neuron shows no modulation in response to RDPs with local dots moving in the antipreferred direction (Fig. $3 A$, squares), indicating high direction selectivity. In contrast, Figure $3 B$ shows the responses of another example neuron that has been excluded from analysis. The responses to preferred motion RDPs show no position-dependent increase and are consequently not well fit by the Gaussian model (Fig. 3B, thick lines). Furthermore, responses to antipreferred motion RDPs (Fig. $3 B$, squares and thin lines) largely overlap the responses to preferred motion RDPs (Fig. $3 B$, circles). These results indicate that the RDPs did not cross the excitatory RF region.

We validated our selection procedure at the level of the population by computing a DSI for each neuron within each response bin and averaging those across the neuronal population (Fig. 3C). The average DSI of the group of neurons selected for further 
analysis (Fig. 3C, solid line) shows a strong increase in direction selectivity along the trajectory of the RDPs while the DSIs of the excluded group (Fig. 3C, dashed line) remain low. A two-way ANOVA confirmed that both groups show different degrees of direction selectivity across the spatial dimension $(p<$ $0.0001)$. The results indicate that our selection process is valid and that the responses of the neurons included in the analysis are modulated by the RDPs position and motion direction. A total of 94 neurons (Monkey Lu, 66; Monkey Se, 28) met this criterion. Of those, 70 matched the criterion in the outward condition (Monkey Lu, $n=49$; Monkey Se, $n=21$; average $R^{2} \pm \mathrm{SD}$ : Attend-Fixation, $0.83 \pm$ 0.14; Tracking, $0.9 \pm 0.09)$, and 64 in the inward condition (Lu, $n=42$; Se, $n=22$; average $R^{2} \pm \mathrm{SD}$ : Attend-Fixation, $0.76 \pm 0.17$; Tracking, $0.84 \pm$ $0.14)$.

To quantify whether the RDPs evoke responses at larger distances from the RF center during Tracking than during AttendFixation, we defined for each neuron the extent of the RF excitatory region as the distance between the center of the Gaussian measured during Attend-Fixation and the point where the predicted values reached half of the Attend-Fixation curve height $\left(\mathrm{DFR}_{50}\right.$; see Materials and Methods, above). The example neurons shown in Figure $4, A$ and $B$, show larger $D_{F R}$ during Tracking than during Attend-Fixation (Tracking: $5.7^{\circ}$, Attend-Fixation: $4.1^{\circ}$; Fig. $4 A$; Tracking: $3.6^{\circ}$, Attend-Fixation: $2.5^{\circ}$; Fig. $4 B$ ).

Figure $4 C$ contrasts the $D^{2} R_{50}$ obtained during AttendFixation (abscissa) with Tracking (ordinate) for each individual neuron and for both translating directions (outward: white squares; inward: gray circles). The majority of the data points fall above the unity line, indicating that the $\mathrm{DFR}_{50}$ is larger during Tracking than during Attend-Fixation. To further quantify this result, we expressed the $\mathrm{DFR}_{50}$ during Tracking relative to the $\mathrm{DFR}_{50}$ during Attend-Fixation for each neuron (Fig. $4 \mathrm{D}$ ). The distributions of these percentages show clear shifts toward positive values in both groups (outward, $p=0.00036$; inward, $p<$ 0.0001 ; Wilcoxon signed-rank test), with median shifts of $12.4 \%$ (outward) and $27 \%$ (inward). This result suggests that during Tracking, the stimuli activated the excitatory RF at a larger distance from the RF center relative to fixation.

One possible explanation for this result is that the animals were more alert during Tracking than during Attend-Fixation due to different levels of task difficulty. Although trials were randomly interleaved, the animals may have quickly adjusted their alertness at the beginning of the trial, when the FS changed color. This hypothesis predicts that the responses during Tracking would be stronger than during Attend-Fixation at trial onset, before the RDPs entered the RF.

To test this hypothesis, we analyzed the parameters of the Gaussian fits corresponding to the two conditions. A higher level of alertness during Tracking should lead to an increase in the baseline parameter relative to Attend-Fixation. Figure $5 \mathrm{~A}$ shows the PMI computed using the baseline parameters of the fits in the two conditions for each neuron (Eq. 3). For both inward and outward trials (Fig. 5A, top and bottom, respectively) we found no difference in baseline between conditions (inward: median
A

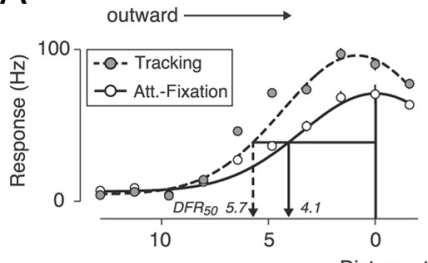

« inward

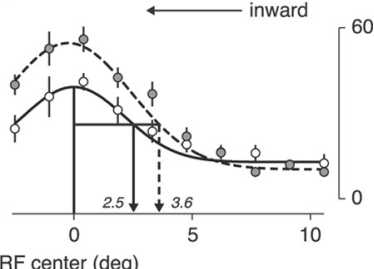

C
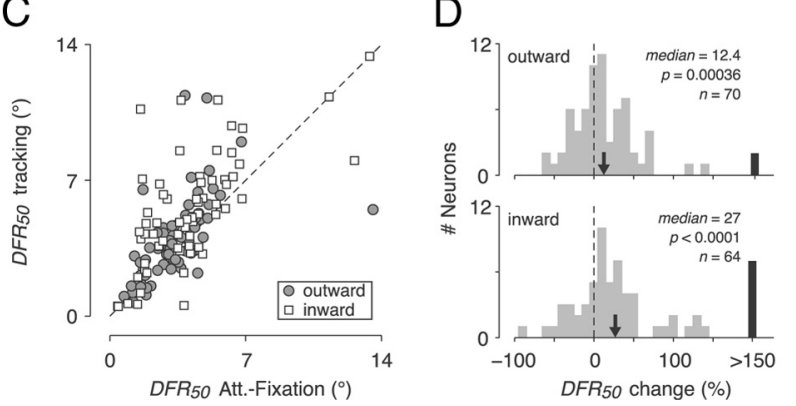

Figure 4. Example result. $\boldsymbol{A}$, Single neuron example corresponding to outward trials (left to right; arrow). (ircles represent the average responses evoked by RDPs with local dots moving in the neuron's preferred direction during Tracking (gray) and Attend-Fixation (white). Gaussian fit predicted values are superimposed (Tracking, dashed line; Attend-Fixation, solid line). The $D \mathrm{FR}_{50}$ (down arrows) represents the distance from the Gaussian center to the point of halfmaximum response during Attend-Fixation (horizontal line). $\boldsymbol{B}$, Single neuron example corresponding to inward trials (right to left). The same conventions as in $\boldsymbol{A}$ apply. $\boldsymbol{C}$, Population analysis. Each data point represents the $\mathrm{DFR}_{50}$ of an individual neuron during Attend-Fixation (abscissa) and Tracking (ordinate) for outward (black circles) and inward (white squares) trials. D, The histograms display the $\mathrm{DFR}_{50}$ during Tracking relative to the $\mathrm{DFR}_{50}$ during AttendFixation. Dark bars summarize number of neurons with $\mathrm{DFR}_{50}$ changes $>150 \%$. The arrows depict the median. Att., Attend.

MI, $-0.009[-1.8 \%], p=0.33$; outward: median MI, -0.03 $[-5.8 \%], p=0.19$; Wilcoxon signed-rank test). This result suggests that increases in firing rate due to changes in the animals' alertness did not cause the observed changes in response.

One possible explanation for the observed effects is that, relative to Attend-Fixation, the effective size of the RF remained the same during Tracking but its entire area shifted toward the approaching patterns. To test this, we compared the location of the RF center responses (parameter $C$ of the Gaussian fit) between both conditions (Fig. 5B). We found that neither the MIs corresponding to inward trials (median MI: $0.005[1 \%], p=0.38$, 


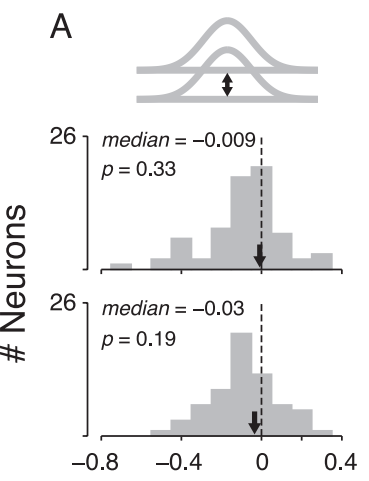

B

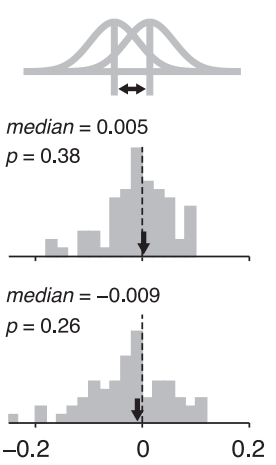

C

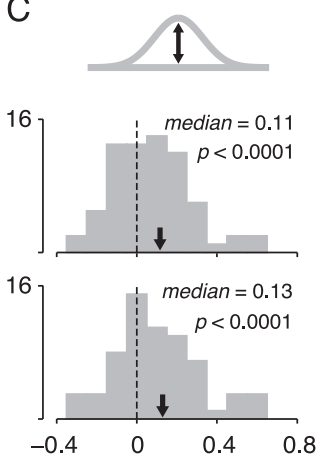

$\mathrm{F}$
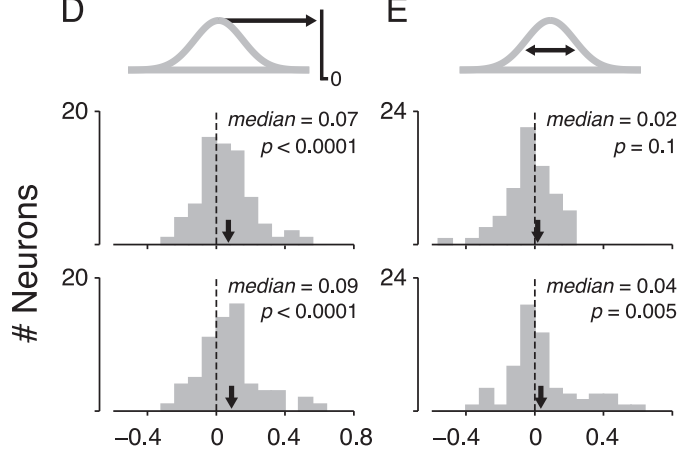

\section{PMI}

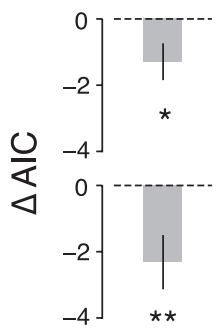

$\begin{array}{lll}-0.4 & 0 & 0.4\end{array}$

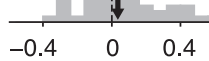

Figure 5. Analysis of fitting parameters. $\boldsymbol{A}-\boldsymbol{F}$, The histograms show the modulation indices of fitting parameters (PMI) and the maximum response for all neurons of the outward (top) and inward (bottom) group. The arrows indicate the median PMI. The icons on top indicate what each PMI measures. $\boldsymbol{A}$, Baseline. $\boldsymbol{B}$, Peak location. $\boldsymbol{C}$, Height. $\boldsymbol{D}$, Maximum response. $\boldsymbol{E}$, Width. $\boldsymbol{F}$, Difference in AlC for two different Gaussian models fitted to the Tracking data. ${ }^{*} p=0.023,{ }^{* *} p=0.006, t$ test.

Wilcoxon signed-rank test) nor those corresponding to outward trials (median MI: $-0.009[-1.8 \%], p=0.26$; Wilcoxon signedrank test) showed a significant shift in the center parameter between the two conditions.

One observation in the previous single neuron example was that the responses close to the center of the RF were larger during Tracking than during Attend-Fixation. A parameter of the Gaussian function that should capture these response changes is the height of the curve, i.e., the difference between a neuron's baseline response and the response when RDPs cross the RF center. Indeed, the MIs of both the outward (median MI: 0.11 [25\%], $p<0.0001$; Wilcoxon signed-rank test) and inward (median MI: $0.13[30 \%], p<0.0001$; Wilcoxon signed-rank test) group are strongly positive, reflecting increases in the neurons' response at the RF center during Tracking (Fig. 5C). Together with the effects isolated in Figure 4, these data suggest that the neurons signaled the presence of the stimulus with stronger firing patterns during Tracking compared with Attend-Fixation.

Although the previous analysis of the baseline parameter showed that Tracking has no effect on baseline activity, a mild reduction in baseline firing rate could, in theory, have led to the observed increases in the parameter height even if the neurons peak response remained unchanged. We tested this hypothesis by comparing the absolute peak responses obtained in both conditions (Fig. 5D). The MIs in both the outward (median MI: 0.07 $[15 \%], p<0.0001$; Wilcoxon signed-rank test) and inward (median MI: 0.09 [19.8\%], $p<0.0001$; Wilcoxon signed-rank test) groups are strongly positive, indicating that the peak responses were higher during Tracking. This demonstrates that the previously reported increase in height is due to higher neuronal firing rates during Tracking rather than to baseline reductions.
One important question arising from our results is whether the observed RF expansion is due to a multiplication of the Gaussian RF profile during Tracking relative to Attend-Fixation. This hypothesis predicts an increase in the parameter height without changes in width during the former relative to the latter condition. However, a nonlinear expansion of the RF profile would predict that the width increased during Tracking. We found that modulation indices between Tracking and Attend-Fixation corresponding to the parameter $W$ (Fig. $5 E$ ) were not significantly different in the outward group (median MI: 0.02 [4.1\%], $p=0.1$; Wilcoxon signed-rank test), but were significantly larger than zero in the inward group (median MI: 0.04 [8.3\%], $p=$ 0.005; Wilcoxon signed-rank test). To further confirm this result, we repeated the $\mathrm{DFR}_{50}$ analysis; however, unlike in the previous analysis, here the $\mathrm{DFR}_{50}$ was determined with respect to the center of the Gaussian fit of each condition separately. The results are similar to those obtained in the analysis of the parameter $W$. Tracking did not cause a significant change in $\mathrm{DFR}_{50}$ of the outward group (median shift: $-4.7 \%, p=0.49$; Wilcoxon signedrank test), while for the inward group it caused a clear shift (median shift: 12.2\%, $p=0.0014$; Wilcoxon signed-rank test).

To further clarify this issue, we conducted an additional analysis. For each neuron, we fitted the Tracking data with two different models. In the first one, all parameters were allowed to vary, but in the second one the width parameter was fixed to the value determined during the Attend-Fixation condition. We then computed the AIC for the two different models. Importantly, this criterion takes into account the number of free parameters in a model, providing an unbiased measurement of the model's goodness of fit that can be used to compare models with different number of parameters. To do the latter, we subtracted the AIC corresponding to both models ( $\triangle \mathrm{AIC}$; see Materials and Methods, above). A negative $\triangle$ AIC indicates that the model in which the width was allowed to vary provides a better fit to the data; a $\triangle$ AIC value not different from zero means that both models equally fit the data and therefore the width parameters does not significantly change during Tracking relative to Attend-Fixation.

We found that for both outward (mean $\Delta$ AIC: $-1.3, p=$ $0.023, t$ test) and inward (mean $\Delta$ AIC: $-2.3, p=0.006, t$ test) groups, the $\triangle \mathrm{AIC}$ was negative, indicating that the fits significantly improved when the width term was allowed to vary (Fig. $5 F$ ). This result suggests that the width of the RF profile changed during Tracking, and thus that the observed increase in response across the RF regions was not proportional.

To determine whether the previously isolated changes in width were due to artifacts in the data analysis introduced by the fitting procedure, we conducted a different test directly comparing the firing rates of the neurons. Figure $6 \mathrm{~A}$ depicts the responses of two example neurons during Tracking (dashed lines) and Attend-Fixation (solid lines). The firing rates in the two conditions appear to diverge more strongly before the maximum 
A
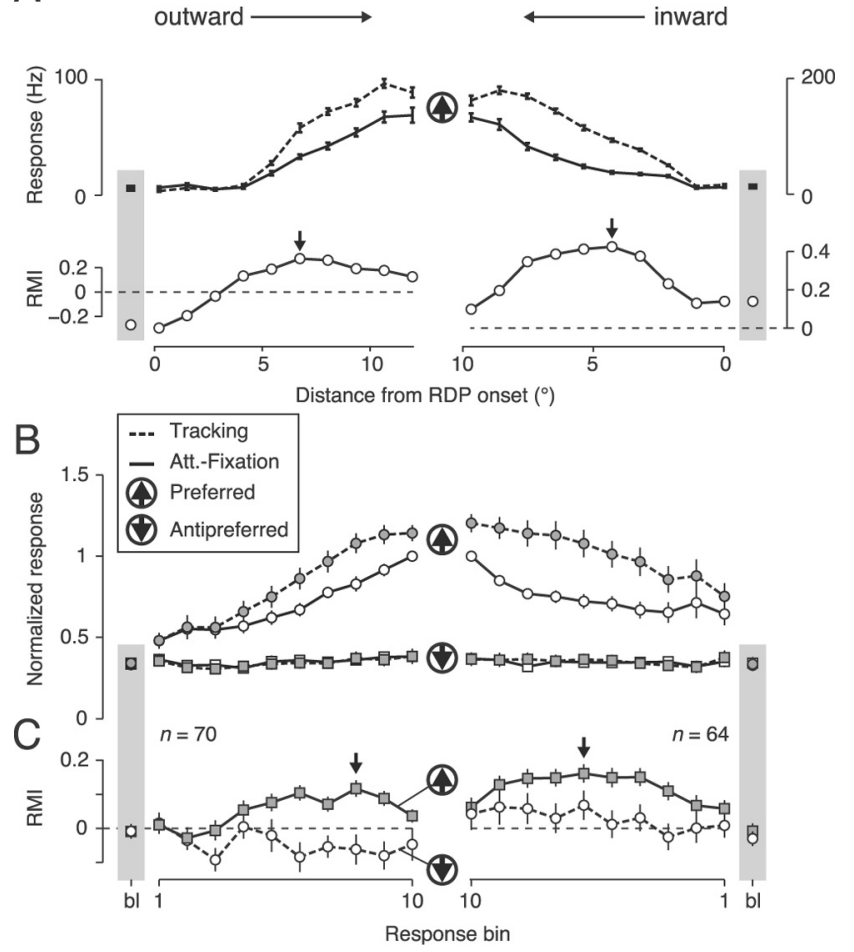

Figure 6. Nonlinear RF expansion. $\boldsymbol{A}$, Single neuron example responses (top) evoked by preferred direction RDPs during Tracking (dashed line) and Attend-Fixation (solid line), and the corresponding RMIs (bottom). The arrow indicates the position of the strongest modulation. $\boldsymbol{B}$, Population responses. The data represent the average responses evoked by preferred (circles) and antipreferred (squares) RDPs. C, Population RMls computed on responses evoked by preferred (squares) and antipreferred (circles) direction RDPs. The arrows depict the bin where the strongest average modulation occurred. bl, Baseline response without the stimulus. Error bars indicate SEM. Att., Attend.

firing rate in the Attend-Fixation condition (i.e., the putative RF center) is reached. We quantified this effect by computing an RMI for each neuron and response bin along the RDPs trajectory (see Materials and Methods, above). In both examples, RMIs were low when RDPs were outside the RF, increased as they entered the RF, and decreased again as the RDPs reached the RF center. In both cases, the peak attentional modulation occurred at intermediate distances between the position of the RDPs at trial onset and the RF center (Fig. 6A, arrows). The population averages (Fig. $6 \mathrm{~B}$ ) and the population RMIs (Fig. 6C, solid lines) confirm this observation. For both the outward and inward groups, the strength of the modulation changes along the RDPs' trajectory (outward, $p<0.0001$; inward, $p=0.01$; one-way ANOVA), exhibiting peak modulations before the RDPs reach the putative RF center (Fig. 6C, arrows). This result supports the hypothesis that RFs undergo nonlinear changes with larger increases in firing rate at positions away from the center during Tracking.

Using the same method, we tested whether the baseline firing rates differ between conditions. Figure $6 \mathrm{~A}$, gray boxes, shows the average firing rates of the example neurons during the cueing period (590 ms) before the onset of the RDPs. The differences are very small, indicating that the firing rates were not influenced by the cue. On the population level, we found the same results (Fig. $6 \mathrm{~B}$ ). The average firing rates of the two conditions largely overlap and the RMIs (Fig. $6 \mathrm{C}$ ) are not significantly different from zero (outward: mean RMI, $-0.009[-1.8 \%], p=0.65$; inward: mean RMI, $-0.008[-1.6 \%], p=0.73, t$ test). This result agrees with the previous finding of similar baseline parameters in both conditions (Fig. 5A).

Although the lack of increases in baseline during Tracking relative to Attend-Fixation argues against the explanation that the isolated effects were due to the animals adjusting their alertness or level of arousal during a trial, we performed additional tests to discard this hypothesis. We tested the prediction that increases in response due to arousal during Tracking should occur for both directions of the local dots in the translating patterns. This is because a response modulation due to arousal is independent of the stimulus but dependent on the internal state of the animal. We examined the difference in response between Tracking and Attend-Fixation when the dots in the RDPs moved in the antipreferred direction.

The square symbols in Figure $6 B$ represent normalized population responses for the Tracking (gray) and Attend-Fixation (white) conditions with antipreferred RDPs. They are virtually identical between conditions and along the spatial extent of the RF. The RMIs confirm this observation (Fig. 6C, open circles, dashed line). They are similar along the spatiotemporal dimension of the trial (outward: $p=0.54$; inward: $p=0.88$, one-way ANOVA). In contrast, responses evoked by the preferred RDPs (Fig. 6B, solid lines) show a clear increase in response during Tracking that departs from the response during Attend-Fixation and grows larger as the patterns approach the RF center. Since the differences in performance between Tracking trials (for both directions of the RDPs' dots) and Attend-Fixation trials were similar (Fig. 2), we conclude that the differences in response when the patterns moved in the preferred direction were not caused by increases in the animals' arousal during Tracking.

\section{Signal detection analysis}

To test whether the observed expansion of the RF borders had an impact on the neurons' performance at detecting a stimulus in their RFs, we applied signal detection analysis. This analysis not only accounts for differences in mean responses between firing rates but also for the trial-to-trial variability in response to the same stimuli (Thompson et al., 1996). Additionally, it is independent on the Gaussian fit analysis illustrated in Figure 4, since it uses the raw firing rates in individual trials. For each neuron, we computed ROC curves comparing the firing rates during a window when the stimuli appeared outside the RF against the firing rate in successive windows as the RDPs moved across the screen and entered the RF. As a measurement of performance (stimulus detection), we computed the auROC in $1 \mathrm{~ms}$ increments, resulting in quasicontinuous curves for each condition. These data were binned and fitted with a Weibull function. Detection thresholds were defined to be at 0.75 (Thompson et al., 1996) and the corresponding spatial position of the RDPs was used to compute the TDD (see Materials and Methods, above).

Figure $7 \mathrm{~A}$ shows auROC data for two example neurons as a function of the RDP position relative to the RF center. For both neurons and conditions, auROC values increase as the RDPs approach the RF center, indicating that responses become steadily more discernible from baseline. More importantly, both neurons exhibit shorter TDDs during Tracking (Fig. 7A, dashed line) relative to Attend-Fixation (Fig. $7 A$, solid line). To validate this observation at the level of the population, we contrasted the TDDs obtained during both conditions for all neurons that reached the threshold criteria (Fig. $7 B$ ). For both groups (outward, $n=66$, Fig. $7 B$, gray circles; inward, $n=56$, Fig. $7 B$, white squares), the majority of the data fall above the unity line, indicating that TDDs are shorter during Tracking. This effect was 
A

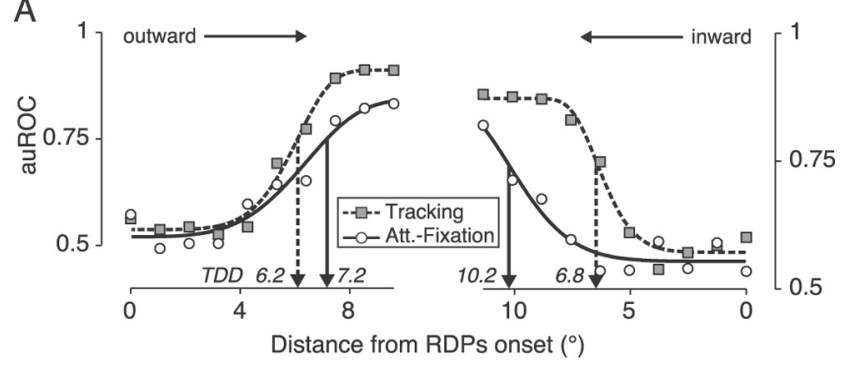

B

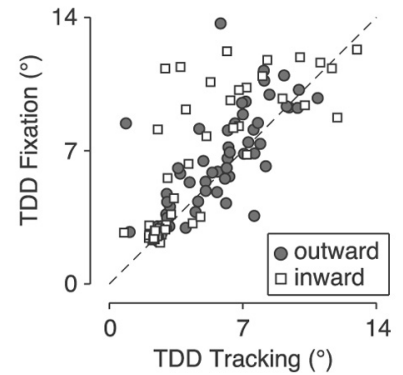

C

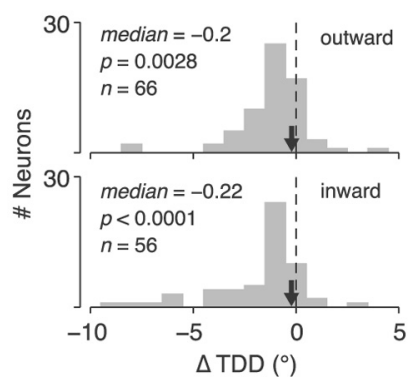

Figure 7. Signal detection analysis. $\boldsymbol{A}$, Left, Example neuron, outward group. Data points represent the auROC (for details, see text) value as a function of the RDPs' position during Attend-Fixation (white circles) and Tracking (gray squares). The data were fitted using Weibull functions (Tracking, dashed line; Attend-Fixation, solid line). Right, Example neuron, inward group. B, Population analysis. Each data point displays TDDs during Attend-Fixation (abscissa) versus Tracking (ordinate) of a neuron. C, Effects of Tracking on TDDs. The histograms display the difference between TDDs during Tracking and Attend-Fixation. Arrows indicate the median. Att., Attend.

further quantified by computing the difference between Tracking and Attend-Fixation TDDs (Fig. 7C). For both groups, the histograms are shifted toward negative values (outward, $p=0.0028$; inward, $p<0.0001$, Wilcoxon signed-rank test), with median shifts of $-0.2^{\circ}$ (outward) and $-0.22^{\circ}$ (inward) and means $-0.64^{\circ}$ (outward) and $-1.2^{\circ}$ (inward). This result suggests that neurons signaled the presence of the RDPs at a shorter distance from the initial RDPs' position, and therefore earlier during Tracking relative to Attend-Fixation.

\section{Fano factor analysis}

Previous studies have reported that attention decreases the variability in neuronal responses, thereby increasing the signal-tonoise ratio corresponding to attended stimuli (Mitchell et al., 2007, 2009; Cohen and Maunsell, 2009). We tested whether the changes in performance we found using ROC analysis were accompanied by a decrease in response variability by computing the Fano factor for each neuron and condition, i.e., the ratio of the variance of the spike counts across trials divided by the mean of the spike counts. Figure $8 A$ shows the Fano factor of two example neurons computed for responses to preferred direction RDPs as a function of the patterns' distance from to their starting position. In both conditions, the Fano factor decreases as the RDPs enter the RFs. More importantly, relative to Attend-Fixation (Fig. $8 \mathrm{~A}$, solid line) the Fano factor during Tracking (Fig. $8 \mathrm{~A}$, dotted line) drops more sharply as the RDPs approach the RF center. This indicates that Tracking reduces the response variability and enhances the signal-to-noise ratio of responses evoked by the RDPs. We found a similar response pattern at the level of the neuronal population (Fig. $8 \mathrm{~B}$ ). Fano factors are similar in both conditions when the RDPs are located outside the RF, but diverge as they approach the RF center. We quantified this effect by computing an FFMI (Fig. 8C). For both translating directions, the FFMIs
A

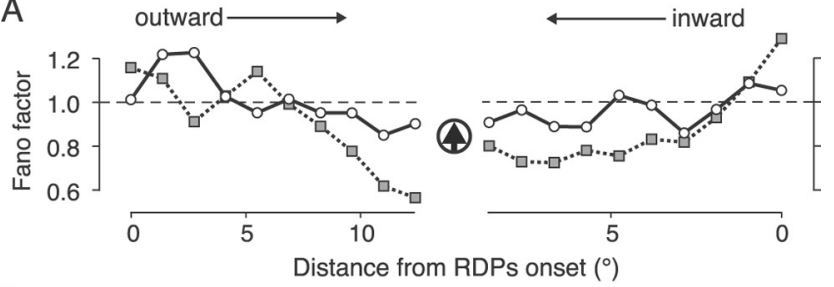

B

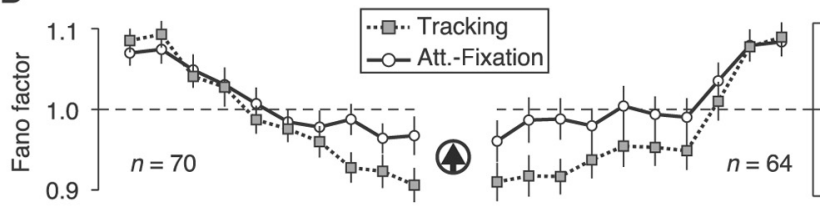

C
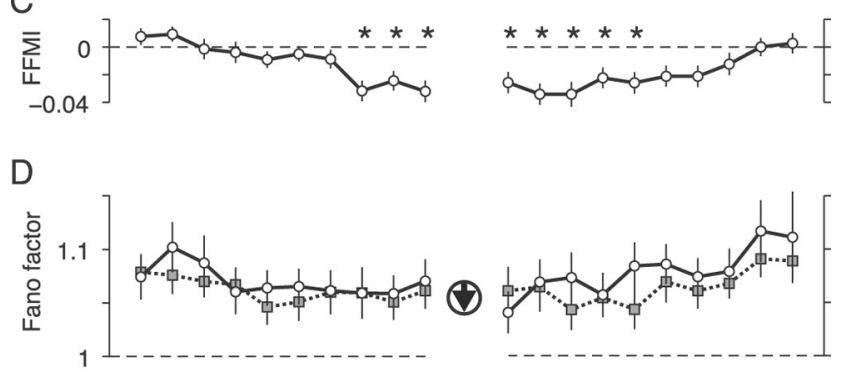

\section{$E$}

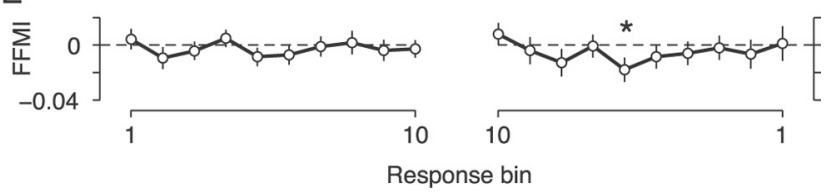

Figure 8. Fano factor analysis. A, Fano factor as a function of the preferred (up arrow) RDPs' position of two example neurons. Dotted line and gray squares represent the Fano factor during Tracking; solid line and white circles represent the Fano factor during Attend-Fixation. $\boldsymbol{B}$, Population averages. C, FFMI corresponding to the data depicted in B. D. Average Fano factor as a function of the antipreferred (down arrow) RDPs' position relative to their onset. $E$, FFMls corresponding to the data depicted in $\boldsymbol{B}$. Error bars indicate SEM. ${ }^{*} p<0.05$, Bonferroni corrected $t$ test. Att., Attend.

decrease along the RDPs trajectories (outward: $p<0.0001$; inward: $p=0.004$; one-way ANOVA), indicating that the signalto-noise ratio progressively increases during Tracking relative to Attend-Fixation.

To test whether the decrease in Fano factor during Tracking also applies to RDPs with local dots moving in the neurons' antipreferred direction, which produced a weak response from the neurons (Churchland et al., 2010), we applied the same analysis. We found a general trend for the Fano factor magnitude to decrease as the RDPs move inside the RF, and to be smaller during Tracking than during fixation (Fig. $8 D$ ). However, the FFMIs do not show a significant modulation as the RDPs enter the RF (outward: $p=0.88$; inward: $p=0.81$; one-way ANOVA; Fig. $8 E$ ). We found that the FFMI became significantly lower than zero for only one data point in the inward dataset when using parametric statistics ( $p<0.05$, Bonferroni corrected $t$ test). These results are compatible with those reported by Churchland et al. (2010) and suggest that the more effective preferred stimuli produce a more robust decrease in response variability during Tracking.

The results of the Fano factor analysis for the RDPs moving in the preferred direction, together with those appearing in Figure 6, indicate that the improvement in signal detection during Tracking is due to both an increase in firing rate and a decrease in response variability. 
A

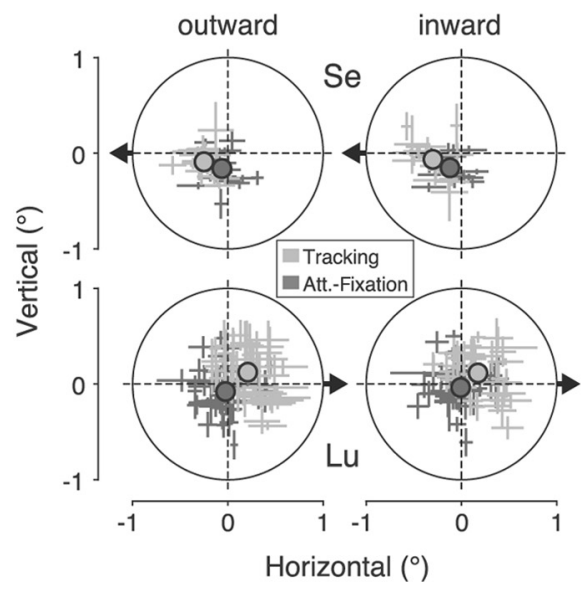

B

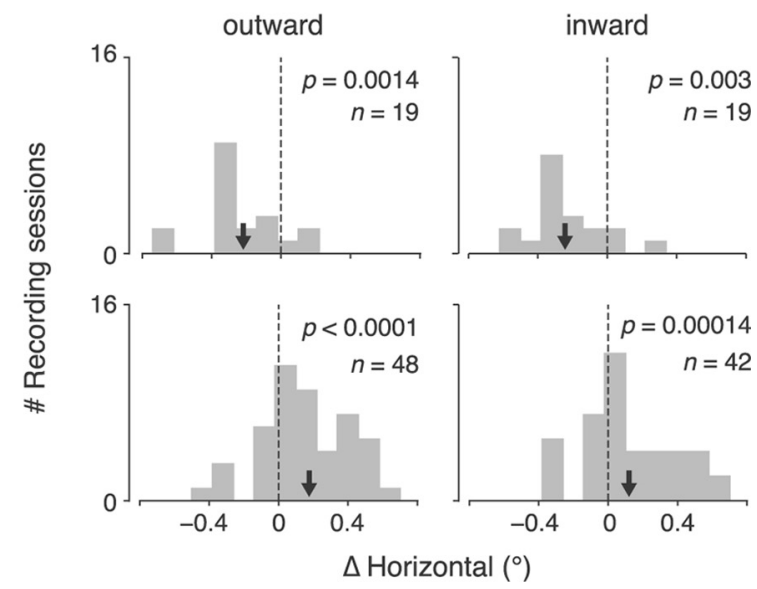

Figure 9. Eye position analysis. $\boldsymbol{A}$, Each data point represents the average eye position ( \pm SD) during Attend-Fixation (dark gray) and Tracking (light gray) in one recording session. Data are aligned to the RF center (arrows). Average deviations across all sessions are indicated by the small circles. The large circles represent the fixation window ( $1^{\circ}$ radius). $\boldsymbol{B}$, Distribution of differences in horizontal eye positions between Tracking and Attend-Fixation. Arrows represent the median. Att., Attend.

\section{Eye position analysis}

Finally, we tested whether the isolated differences in response could be explained by systematic deviations in eye positions between the two conditions. For each session, we calculated the mean eye positions within the $1^{\circ}$ fixation window across trials during both Tracking and Attend-Fixation (Fig. 9A). The data were rotated around the fixation point, so that the RF centers of all recorded neurons were aligned with the axis parallel to the RDPs' trajectories (Fig. 9A, arrows). During Attend-Fixation, the average eye positions appear closer to the fixation spot center [monkey Se: outward (mean distance $\pm \mathrm{SD}$ ): $0.27^{\circ} \pm 0.13^{\circ}$; inward: $0.26^{\circ} \pm 0.1^{\circ}$; monkey Lu: outward: $0.27^{\circ} \pm 0.15^{\circ}$; inward: $0.27^{\circ} \pm 0.15^{\circ}$, while during Tracking they appear slightly shifted in the direction of the RF (monkey Se: outward: $0.31^{\circ} \pm 0.1^{\circ}$; inward: $0.37^{\circ} \pm 0.13^{\circ}$; monkey Lu: outward: $0.37^{\circ} \pm 0.15^{\circ}$; inward: $0.35^{\circ} \pm 0.15^{\circ}$ ). Figure $9 B$ shows the distributions of differences between mean horizontal eye positions in both conditions. The deviations from the fixation spot are larger during Tracking (monkey Se: outward, median difference: $-0.22^{\circ}, p=0.0015$, inward: $-0.24, p=0.003$; monkey Lu: outward: $0.18, p<0.0001$, inward: $0.12, p=0.00014$; Wilcoxon signed-rank test).

Since the RFs of MT neurons are retinotopically organized (Born and Bradley, 2005), systematic shifts in eye position must have different implications for outward and inward trials. For example, during inward trials, they would cause RDPs to reach the RF earlier during Tracking than during Attend-Fixation. Conversely, during outward trials, the shift would cause the opposite effect: RDPs must reach the RF later during Tracking than during Attend-Fixation. We tested whether the shifts in eye position could explain the relative expansion of the RF borders, taking into account the relationship between retinotopy and translation direction. For outward trials, we added the difference in horizontal eye position of individual recording sessions to the effects isolated in the $\mathrm{DFR}_{50}$ and ROC analysis of each corresponding neuron and recomputed their magnitude. For inward trials, we subtracted the differences in eye position from the neuronal effects. We did not find qualitative changes in the patterns of the results reported previously $\left(\mathrm{DFR}_{50}\right.$ : median shift outward, 21.7\%, $p<0.0001$; median shift inward, 20.6\%, $p<0.0001$; ROC: median $\Delta$ TDD outward, $-0.48^{\circ}, p<0.0001$, median $\Delta$ TDD inward, $-0.25^{\circ}, p=0.0031$; Wilcoxon signed-rank test). These results confirm that the isolated effects are not caused by differences in eye position between the two conditions. However, taking the eye position effects into account decreases the magnitude of the differences between inward and outward trials.

\section{Discussion}

Our study explored the responses of single neurons in area MT of macaques during two different tasks that required either tracking two translating stimuli with covert attention, or attending to a stimulus positioned at the center of gaze while ignoring the translating stimuli. Our results show that MT neurons increase their responses to tracked stimuli passing across their RF relative to responses when the same stimuli are behaviorally irrelevant. This effect is larger at the RF periphery than at its center. Moreover, the variability of the neurons response (Fano factor) became smaller as a tracked stimulus approaches the RF center relative to when the same stimulus is ignored. These changes ultimately improve the neurons' detection performance.

One theoretically possible explanation for our pattern of results is that the animals had different levels of arousal/attentional effort during Tracking relative to Attend-Fixation trials (i.e., performance during Attend-Fixation was significantly higher than during Tracking) (Spitzer et al., 1988; Boudreau et al., 2006; Chen et al., 2008). In our experiments, at least three factors argue against this explanation. First, during experimental sessions, all trial types were randomly interleaved; thus, the animals could not anticipate the level of difficulty of the oncoming trial and adjust their attentional effort accordingly. Second, we did not find systematic increases in response during Tracking relative to Attend-Fixation before the RDPs entered the RF. Third, we did not find differences in neuronal responses between the two conditions when the RDPs moved in the antipreferred direction. An increase in arousal/attentional effort during Tracking relative to Attend-Fixation predicts that both baseline and antipreferred responses will be larger during the former relative to the latter condition.

\section{Mechanisms underlying the expansion of the RF during tracking}

One possible mechanism underlying the RF expansion isolated in our data is that, in anticipation of a tracked object entering the RF, top-down attentional signals (Moore and Armstrong, 2003; 
Armstrong et al., 2006; Saalmann et al., 2007) decrease the activation threshold of MT neurons to incoming sensory inputs from upstream areas such as V1 (Reynolds et al., 2000). One could model the spatial integration of excitatory inputs by an MT neuron (e.g., from V1 direction-selective neurons with small RFs) as a 2D Gaussian function with more input density at the center and less in the periphery (Born and Bradley, 2005; Rust et al., 2006), and a similar proportion of inputs selective for different motion directions to all RF areas. Under these premises, one could hypothesize that decreasing the threshold for firing action potentials during Tracking would make the MT neuron more sensitive to fewer activated inputs at the edge of the Gaussian profile, resulting in an expansion of the RF borders.

A second possibility is that the activation threshold does not change during Tracking for a given MT neuron. Rather, what may change is the strength of the incoming excitatory inputs due to an increase in the responses of upstream neurons (e.g., in V1) that project to the recipient MT neuron. This mechanism has been proposed in previous studies of attention to explain the modulation of responses to two stimuli inside the RF of MT and V4 neurons (Treue and Maunsell, 1999; Ghose and Maunsell, 2008; Ghose, 2009; Khayat et al., 2010a,b). A similar mechanism has been proposed to explain shifts in MT neurons RF toward an attended stimulus (Compte and Wang, 2006; Womelsdorf et al., 2006). A finding that may argue in favor of this input modulation over the single sensitivity/threshold modulation is that the increases in response during Tracking were proportionally larger at the RF periphery than at its center. This nonlinearity is difficult to explain by a single change in the MT neurons' activation threshold. However, for the case of the input modulation, the nonlinearity could be obtained by differentially changing the strength of inputs coming from V1 neurons with RFs corresponding to the periphery (larger gain) and center (smaller gain) of the recipient MT neuron RF. Such input changes may produce inhomogeneities in divisive normalization across different RF regions. The latter idea agrees with models proposing that attention acts by changing the strength of response normalization mechanisms in visual cortex (Ghose, 2009; Lee and Maunsell, 2009; Reynolds and Heeger, 2009).

Further supporting the input modulation hypothesis, the RF expansion was mainly observed for RDPs with local dots moving in the neurons' preferred direction. Models of how MT neurons transform inputs into a response propose that the contribution of inputs tuned for the preferred direction to a neurons' activation is large while the contribution if inputs tuned for the opposite antipreferred direction is small (Simoncelli and Heeger, 1998; Rust et al., 2006). Thus, increases in the activation strength of inputs tuned for the preferred direction would increase the response. In contrast, increases in the activation of inputs tuned for the antipreferred direction would have little effect on the neurons' response (Khayat et al., 2010). Another possibility that can explain this finding is that feature-based attention decreases the response of an MT neuron when its antipreferred direction is attended, counterbalancing the response increase caused by directing attention into the neuron's RF (Martinez-Trujillo and Treue, 2004) and yielding no modulation of the neuron's firing rate.

We found that during Tracking, some neurons show a small decrease in the Gaussian baseline parameter relative to AttendFixation. One possible explanation for this effect is that during Tracking, the patterns entered the RF inhibitory surround before crossing the excitatory border, producing a small decrease in the baseline response (Sundberg et al., 2009). Although in our study we did not have a stimulus in the RF, which is usually needed to reveal center surround interactions (Huang et al., 2008), it is possible that a small amount of inhibition in baseline firing rates occurred. Nevertheless, such an effect had no repercussions in the neurons' ability to detect the stimuli and was largely masked by the expansion of the excitatory RF.

A puzzling finding in our dataset is that, although the changes in response were larger at positions away from the RF center, the decrease in response variability during tracking (i.e., decrease in Fano factor) was progressively larger as the RDPs moved toward the RF center. The interpretation of this result is not trivial and could mean that increases in response during attentive tracking do not necessarily map into proportional decreases in response variability (McAdams and Maunsell, 1999). We found that smaller increases in response at the RF center can still translate into larger decreases in variability relative to the periphery. This may be a consequence of response normalization mechanisms, wherein increases in input summation and therefore in normalization strength may translate into smaller response enhancements (Reynolds and Heeger, 2009) but less variable firing rates. This issue needs to be further investigated.

\section{Origins of the modulatory signal}

The top-down signal that produced the observed changes in response during tracking may originate in saccade-related structures such as frontal eye field (FEF) (Moore and Fallah, 2001) or the superior colliculus (Cavanaugh and Wurtz, 2004). It is possible that during tracking, signals related to saccade preparation reach MT neurons activated by the RDPs (Barborica and Ferrera, 2003; Cassanello et al., 2008). Favoring this hypothesis, it has been reported that FEF neurons are active during attentive tracking (Xiao et al., 2007) and that microstimulation of the FEF produces changes in the RF profile of V4 neurons (Armstrong et al., 2006). The small differences between the magnitude of the effects in outward and inward trials may relate to biases in eye movement planning toward concentrically and eccentrically moving targets (Segraves et al., 1987; Xiao et al., 2006). One issue to be addressed by future studies is whether changes in the RF or movement field profiles of FEF neurons precede the expansion of MT RFs described in our study.

Finally, our results provide neuronal correlates of previously reported increases in ERPs and BOLD-signal amplitude during tracking (Culham et al., 1998; Drew et al., 2009; Howe et al., 2009; Doran and Hoffman, 2010). Furthermore, they support behavioral reports linking attention and tracking performance (Yantis, 1992; Sears and Pylyshyn, 2000; Cavanagh and Alvarez, 2005; Tombu and Seiffert, 2008; Iordanescu et al., 2009; Niebergall et al., 2010). We propose that the expansion of the RF border accompanied by increases in signal-to-noise ratio in MT neurons during attentive tracking (McAdams and Maunsell, 1999; Cohen and Newsome, 2009) produce increases in the neurons' detection performance that translate into improvements in the organism's ability to track visual targets.

In summary, our findings demonstrate that neuronal RFs in area MT can effectively expand to improve the ability of neurons to encode the features of behaviorally relevant stimuli during tracking. The similarity between the effects of attentive tracking on MT neurons' responses isolated here and those observed when attending to stationary targets by previous studies demonstrate a highly adaptive attentional mechanism that allows the primate visual system to cope with a variety of task demands.

\section{References}

Akaike H (1974) A new look at the statistical model identification. IEEE

Trans Auto Contr 19:716-723. 
Anton-Erxleben K, Stephan VM, Treue S (2009) Attention reshapes centersurround receptive field structure in macaque cortical area MT. Cereb Cortex 19:2466-2478.

Armstrong KM, Fitzgerald JK, Moore T (2006) Changes in visual receptive fields with microstimulation of frontal cortex. Neuron 50:791-798.

Barborica A, Ferrera VP (2003) Estimating invisible target speed from neuronal activity in monkey frontal eye field. Nat Neurosci 6:66-74.

Born RT, Bradley DC (2005) Structure and function of visual area MT. Annu Rev Neurosci 28:157-189.

Boudreau CE, Williford TH, Maunsell JH (2006) Effects of task difficulty and target likelihood in area V4 of macaque monkeys. J Neurophysiol 96:2377-2387.

Cassanello CR, Nihalani AT, Ferrera VP (2008) Neuronal responses to moving targets in monkey frontal eye fields. J Neurophysiol 100:1544-1556.

Cavanagh P, Alvarez GA (2005) Tracking multiple targets with multifocal attention. Trends Cogn Sci 9:349-354.

Cavanaugh J, Wurtz RH (2004) Subcortical modulation of attention counters change blindness. J Neurosci 24:11236-11243.

Chen Y, Martinez-Conde S, Macknik SL, Bereshpolova Y, Swadlow HA, Alonso JM (2008) Task difficulty modulates the activity of specific neuronal populations in primary visual cortex. Nat Neurosci 11:974-982.

Churchland MM, Yu BM, Cunningham JP, Sugrue LP, Cohen MR, Corrado GS, Newsome WT, Clark AM, Hosseini P, Scott BB, Bradley DC, Smith MA, Kohn A, Movshon JA, Armstrong KM, Moore T, Chang SW, Snyder LH, Lisberger SG, Priebe NJ, et al. (2010) Stimulus onset quenches neural variability: a widespread cortical phenomenon. Nat Neurosci 13: 369-378.

Cohen MR, Maunsell JH (2009) Attention improves performance primarily by reducing interneuronal correlations. Nat Neurosci 12:1594-1600.

Cohen MR, Newsome WT (2009) Estimates of the contribution of single neurons to perception depend on timescale and noise correlation. J Neurosci 29:6635-6648.

Compte A, Wang XJ (2006) Tuning curve shift by attention modulation in cortical neurons: a computational study of its mechanisms. Cereb Cortex 16:761-778.

Connor CE, Gallant JL, Preddie DC, Van Essen DC (1996) Responses in area V4 depend on the spatial relationship between stimulus and attention. J Neurophysiol 75:1306-1308.

Culham JC, Brandt SA, Cavanagh P, Kanwisher NG, Dale AM, Tootell RB (1998) Cortical fMRI activation produced by attentive tracking of moving targets. J Neurophysiol 80:2657-2670.

Doran MM, Hoffman JE (2010) The role of visual attention in multiple object tracking: evidence from ERPs. Atten Percept Psychophys 72:33-52.

Drew T, McCollough AW, Horowitz TS, Vogel EK (2009) Attentional enhancement during multiple-object tracking. Psychon Bull Rev 16:411-417.

Ghose GM (2009) Attentional modulation of visual responses by flexible input gain. J Neurophysiol 101:2089-2106.

Ghose GM, Maunsell JH (2008) Spatial summation can explain the attentional modulation of neuronal responses to multiple stimuli in area V4. J Neurosci 28:5115-5126.

Howe PD, Horowitz TS, Morocz IA, Wolfe J, Livingstone MS (2009) Using fMRI to distinguish components of the multiple object tracking task. J Vis 9:10.1-10.11.

Huang X, Albright TD, Stoner GR (2008) Stimulus dependency and mechanisms of surround modulation in cortical area MT. J Neurosci 28:13889-13906.

Iordanescu L, Grabowecky M, Suzuki S (2009) Demand-based dynamic distribution of attention and monitoring of velocities during multiple-object tracking. J Vis 9:1.1-1.12.

Khayat PS, Niebergall R, Martinez-Trujillo JC (2010a) Attention differentially modulates similar neuronal responses evoked by varying contrast and direction stimuli in area MT. J Neurosci 30:2188-2197.

Khayat PS, Niebergall R, Martinez-Trujillo JC (2010b) Frequency-dependent attentional modulation of local field potential signals in macaque area MT. J Neurosci 30:7037-7048.

Lee J, Maunsell JH (2009) A normalization model of attentional modulation of single unit responses. PLoS One 4:e4651.
Martinez-Trujillo JC, Treue S (2004) Feature-based attention increases the selectivity of population responses in primate visual cortex. Curr Biol 14:744-751.

McAdams CJ, Maunsell JH (1999) Effects of attention on the reliability of individual neurons in monkey visual cortex. Neuron 23:765-773.

Mitchell JF, Sundberg KA, Reynolds JH (2007) Differential attentiondependent response modulation across cell classes in macaque visual area V4. Neuron 55:131-141.

Mitchell JF, Sundberg KA, Reynolds JH (2009) Spatial attention decorrelates intrinsic activity fluctuations in macaque area V4. Neuron 63:879-888.

Moore T, Armstrong KM (2003) Selective gating of visual signals by microstimulation of frontal cortex. Nature 421:370-373.

Moore T, Fallah M (2001) Control of eye movements and spatial attention. Proc Natl Acad Sci U S A 98:1273-1276.

Moran J, Desimone R (1985) Selective attention gates visual processing in the extrastriate cortex. Science 229:782-784.

Niebergall R, Huang L, Martinez-Trujillo JC (2010) Similar perceptual costs for dividing attention between retina- and space-centered targets in humans. J Vis 10:4.

Reynolds JH, Heeger DJ (2009) The normalization model of attention. Neuron 61:168-185.

Reynolds JH, Chelazzi L, Desimone R (1999) Competitive mechanisms subserve attention in macaque areas V2 and V4. J Neurosci 19:1736-1753.

Reynolds JH, Pasternak T, Desimone R (2000) Attention increases sensitivity of V4 neurons. Neuron 26:703-714.

Rust NC, Mante V, Simoncelli EP, Movshon JA (2006) How MT cells analyze the motion of visual patterns. Nat Neurosci 9:1421-1431.

Saalmann YB, Pigarev IN, Vidyasagar TR (2007) Neural mechanisms of visual attention: how top-down feedback highlights relevant locations. Science 316:1612-1615.

Sears CR, Pylyshyn ZW (2000) Multiple object tracking and attentional processing. Can J Exp Psychol 54:1-14.

Segraves MA, Goldberg ME, Deng SY, Bruce CJ, Ungerleider LG, Mishkin M (1987) The role of striate cortex in the guidance of eye movements in the monkey. J Neurosci 7:3040-3058.

Seidemann E, Newsome WT (1999) Effect of spatial attention on the responses of area MT neurons. J Neurophysiol 81:1783-1794.

Simoncelli EP, Heeger DJ (1998) A model of neuronal responses in visual area MT. Vision Res 38:743-761.

Spitzer H, Desimone R, Moran J (1988) Increased attention enhances both behavioral and neuronal performance. Science 240:338-340.

Sundberg KA, Mitchell JF, Reynolds JH (2009) Spatial attention modulates center-surround interactions in macaque visual area v4. Neuron 61:952-963.

Thompson KG, Hanes DP, Bichot NP, Schall JD (1996) Perceptual and motor processing stages identified in the activity of macaque frontal eye field neurons during visual search. J Neurophysiol 76:4040-4055.

Tombu M, Seiffert AE (2008) Attentional costs in multiple-object tracking. Cognition 108:1-25.

Treue S, Martinez-Trujillo JC (1999) Feature-based attention influences motion processing gain in macaque visual cortex. Nature 399:575-579.

Treue S, Maunsell JH (1996) Attentional modulation of visual motion processing in cortical areas MT and MST. Nature 382:539-541.

Treue S, Maunsell JH (1999) Effects of attention on the processing of motion in macaque middle temporal and medial superior temporal visual cortical areas. J Neurosci 19:7591-7602.

Womelsdorf T, Anton-Erxleben K, Pieper F, Treue S (2006) Dynamic shifts of visual receptive fields in cortical area MT by spatial attention. Nat Neurosci 9:1156-1160.

Xiao Q, Barborica A, Ferrera VP (2006) Radial motion bias in macaque frontal eye field. Vis Neurosci 23:49-60.

Xiao Q, Barborica A, Ferrera VP (2007) Modulation of visual responses in macaque frontal eye field during covert tracking of invisible targets. Cereb Cortex 17:918-928.

Yantis S (1992) Multielement visual tracking: attention and perceptual organization. Cognit Psychol 24:295-340. 